\title{
Author Correction: Robust topologically protected transport in photonic crystals at telecommunication wavelengths
}

Mikhail I. Shalaev, Wiktor Walasik, Alexander Tsukernik, Yun Xu and Natalia M. Litchinitser

Correction to: Nature Nanotechnology https://doi.org/10.1038/s41565-018-0297-6 published online 12 November 2018.

In the version of this Letter originally published, Fig. $5 \mathrm{~g}$ in the Supplementary Information was missing the scale bar. This has now been corrected.

Published online: 17 December 2018

https://doi.org/10.1038/s41565-018-0349-y 